\title{
Patient-centred care during the COVID-19 pandemic
}

\section{Protocol for a qualitative collective case study by high-performing general practice teams}

Bryce Brickley, Lauren T Williams, Mark Morgan, Alyson Ross, Kellie Trigger, Lauren Ball

\section{Background and objective \\ The COVID-19 pandemic has changed the way general practice teams interact with patients. This protocol article describes the rationale and design of an exploration of the experiences and perceptions of patient-centred care (PCC) by high-functioning general practice teams in Australia.}

\section{Methods}

A qualitative descriptive approach and collective case study method will be used. Potential participants are individuals representing a high-functioning general practice team, who will be asked to participate in a semi-structured interview. Eligible general practice clinics have received a 'Practice of the Year' award or commendation from The Royal Australian College of General Practitioners or Australian General Practice Accreditation Limited. Interview data will be analysed thematically, with constant comparison and meta-synthesis.

\section{Discussion}

Collective case study research is valuable because it enables rich exploration of PCC within the context of the pandemic. Rapid research on PCC may highlight new approaches towards the delivery of PCC.
THE WORLD HEALTH ORGANIZATION defines patient-centred care (PCC) as care that is respectful and responsive to the needs and wishes of patients. ${ }^{1}$ There are many reported benefits of $\mathrm{PCC}$, including improved healthcare outcomes, ${ }^{2-5}$ enhanced relationships between providers and patients ${ }^{4}$ and enhanced patient satisfaction. ${ }^{2,6}$ There is a clear need to explore the processes and implementation of PCC because of its impact on patient experience, providers and organisations, and its long-standing emphasis within healthcare policy, such as the National Safety and Quality Health Service Standards ${ }^{7}$ in Australia.

The COVID-19 pandemic has brought about sweeping changes to healthcare systems across the world, including in Australia, where physical distancing regulations and public health orders affected the availability of general practice staff and influenced the way patients accessed and interacted with general practice services. ${ }^{8}$ Initially, fewer patients attended general practice services for routine care. ${ }^{8,9}$ Then, general practices were called on to administer COVID-19 vaccinations to a significant proportion of the national population. The COVID-19 pandemic caused a significant disruption to general practice, and its impact on PCC is not yet understood.
Patient centredness is a dynamic concept in global health systems, and it is susceptible to compromise if priorities of healthcare shift, such as in times of a health crisis. Published in 2021, a Delphi study involving 114 healthcare experts, managers, clinicians and patients expressed there may be new priorities in achieving PCC in the COVID-19 era, including greater emphasis on patient safety, care for vulnerable patients, promotion of self-management and patient autonomy. ${ }^{10}$ These priorities diverge from what was known about enacting PCC prior to the pandemic, which focused on six key components: 1 ) understanding the whole person, 2) finding common ground, 3) experiencing time, 4) aiming for positive outcomes, 5) considering the system and collaborating in care and 6) optimising the general practice environment. ${ }^{11}$ Trustworthy qualitative research is valuable to understand the experiences and perspectives of PCC while capturing the contextual factors of the pandemic to inform higher levels of PCC.

The performance of general practice can be externally assessed, and those practices that demonstrate a commitment to quality may be recognised with an award. Award-winning practices are high performing and are considered to have a high-functioning practice team. 
High-functioning general practice teams are the leaders of the workforce and exemplify the characteristics of general practice, which include quality, safety and PCC. ${ }^{12}$ The objective of this study is to explore the attitudes towards, experiences of and perceptions of PCC by Australian high-functioning general practice teams. By aggregating exemplar cases of PCC, the aim of this study is to explore many valuable insights from the experiences and perceptions of PCC within high-performing practices. Rigorous qualitative research on PCC is needed, as the extent of pandemic's influence on PCC is unknown. The identification of novel strategies to deliver PCC throughout the first 18 months of the pandemic is important given PCC is prioritised in the Australian Government's Long Term National Health
Plan..$^{13}$ The proposed research is one of the first studies to explore PCC during the COVID-19 pandemic.

\section{Methods}

\section{Methodological overview}

This study will be situated in a social constructivist philosophical position ${ }^{14}$ to explore PCC in the context of the COVID-19 pandemic. The researchers' view is that all knowledge is constructed by the interaction between human beings and their world, with important influence from the social context. ${ }^{14}$ A qualitative descriptive methodological approach ${ }^{15}$ will be employed to address the research questions, which are outlined in Table 1. This approach is a well-established alternative qualitative methodology that is commonly used to identify the 'who', 'what' and 'where' of experiences in their natural state. This approach is ideal to generate data on the complex concept of PCC in a real-world language ${ }^{15}$ and allow for the identification of PCC strategies that can be easily integrated into practice. The qualitative descriptive approach will also enable a description of PCC that accounts for any influence of the pandemic. ${ }^{15}$

\section{Study design}

The concept of PCC was well defined prior to the COVID-19 pandemic, ${ }^{11,16}$ but the disruption to the general practice sector caused by the pandemic has influenced the social situations in which PCC is delivered. A collective case study method ${ }^{17}$ will be employed to ascertain general practice teams' perspectives of PCC throughout the pandemic and describe PCC initiatives. Brickley and colleagues' map of PCC $^{11}$

\section{Table 1. Semi-structured interview guide}

\begin{tabular}{|c|c|c|c|}
\hline Research questions & Inquiry purpose & Main interview questions & Potential probing questions \\
\hline $\begin{array}{l}\text { What are the attitudes, beliefs } \\
\text { and experiences of PCC by } \\
\text { high-performing general } \\
\text { practices in the context of } \\
\text { the COVID-19 pandemic? }\end{array}$ & $\begin{array}{l}\text { 1. Elicit existing views on PCC } \\
\text { and provide opportunity for } \\
\text { open expression of views } \\
\text { regarding PCC. } \\
\text { 2. Understand the pandemic's } \\
\text { influence on PCC delivery, and } \\
\text { investigate if any attitudinal } \\
\text { changes in practice teams } \\
\text { have taken place. }\end{array}$ & $\begin{array}{l}\text { 1. Can you describe to me your } \\
\text { practice team's views on PCC } \\
\text { throughout the pandemic? } \\
\text { 2. Are there any different views } \\
\text { throughout the practice team? } \\
\text { 3. Have any members of your } \\
\text { practice team's views and } \\
\text { attitudes towards PCC changed } \\
\text { since before the pandemic? } \\
\text { 4. If they have changed, can } \\
\text { you elaborate on how they } \\
\text { are different? If they are the } \\
\text { same, can you explain why? }\end{array}$ & $\begin{array}{l}\text { 1. Do you share the views of your } \\
\text { practice on this topic? } \\
\text { 2. How long have you felt this way? } \\
\text { 3. What advice could you give } \\
\text { another practice striving for PCC } \\
\text { in the current healthcare system? } \\
\text { 4. What do you think the necessary } \\
\text { changes to policy are to promote } \\
\text { PCC in the current environment? } \\
\text { 5. What, if any, are the most effective } \\
\text { models of general practice in } \\
\text { terms of providing PCC? } \\
\text { 6. Further questions to be guided } \\
\text { by findings of initial analysis. }\end{array}$ \\
\hline
\end{tabular}

The Brickley and colleagues map of PCC to be shown to participants at this stage of the interview. All participants are to be given time to read and reflect on the model, with an opportunity to ask any questions.

\begin{tabular}{lcc}
\hline $\begin{array}{l}\text { To what extent have high- } \\
\text { performing general practice }\end{array}$ & $\begin{array}{l}\text { Explore perceived strategies } \\
\text { that the practice has used to }\end{array}$ & $\begin{array}{c}\text { 5. Do you believe that your } \\
\text { practice has been able to }\end{array}$ \\
$\begin{array}{l}\text { staff delivered PCC throughout } \\
\text { sundemic? }\end{array}$ & $\begin{array}{l}\text { of PCC. } \\
\text { What, if any, strategies have }\end{array}$ & 4. Allow participants time to \\
been used to achieve this? & freely express their thoughts & throughout pandemic? \\
& and feelings. & initiatives in your practice \\
& & undertaken towards promoting \\
& & $\begin{array}{l}\text { the delivery of PCC? Can you } \\
\text { describe these? }\end{array}$ \\
& & 7. Is there anything you would like \\
& to express that has not come up \\
& in this interview so far?
\end{tabular}

7. Can you elaborate on the roles of staff in this initiative?

8. What advice could you give another practice intending to undertake the same initiative?

9. Further questions to be guided by findings of initial analysis. 
will inform a foundational descriptive theory, which will be used to guide data collection and analysis, and this is routine in descriptive case study research. The proposed study is collective in design (aggregating cases and synthesising results across participants); it will also be instrumental as participants will be recruited on the basis of their ability to deliver PCC at high levels. Semi-structured interviews will be conducted to identify the experiences and perspectives of PCC. Ethics approval was obtained from the Griffith University Human Research Ethics Committee (No. 2021/132).

\section{Sample and recruitment}

The sampling strategy will aim to recruit information-rich cases to describe PCC as it appears in practices that the researchers believe delivered PCC at high levels prior to the pandemic. ${ }^{15}$ The Royal Australian College of General Practitioners (RACGP) and Australian General Practice Accreditation Limited (AGPAL) provide awards and commendations to general practices throughout the country for quality care. To be eligible for the present study, general practices will have been 1) awarded 'General Practice of the Year' by the RACGP, 2) awarded 'General Practice of the Year' by AGPAL and Quality Innovation Performance Limited (QIP) or 3) highly commended in the 'General Practice of the Year' award by AGPAL and QIP. The identification of high-performing practice has been completed similarly elsewhere. ${ }^{18}$ Practices must have received an award in the past five years to increase confidence of practice high performance.

The RACGP is the leading representative body for general practice in Australia, while AGPAL and QIP are providers of accreditation and quality improvement for general practices. To be awarded, practices must have been accredited, and any two persons must have submitted a nomination of the practice to the relevant organisation with a description of what differentiates the practice from other practices. The 'General Practice of the Year' award signifies quality, safety and best-practice general practice with evidence of continuous quality improvements in terms of efficiencies, processes, staff and patient engagement.

A total of 10 practices were identified from a publicly available list of the respective award winners dating back to 2016, with the most recent award provided in 2020 . The 10 practices will be approached to participate in an interview via telephone by an experienced GP who is also an academic (MM). The practice contact person will be asked to nominate a practice representative to participate in the interview; the representative must have been employed at the practice before the pandemic and willing to speak on behalf of the practice team. The primary researcher will then follow up all nominated interviewees via email with a study flyer and study information sheet and schedule the interview. A representative was chosen rather than a focus group for pragmatic reasons, to not overload general practice teams during the additional demands of pandemic conditions. The interviews will be conducted over telephone or videoconference (Microsoft Teams) and conducted independently by a primary care researcher/dietitian (BB). Participating practices will be paid a $\$ 100$ honorarium.

\section{Interview protocol}

Semi-structured interview questions were informed by an interview guide developed by the research team (Table 1 ). The interview guide was reviewed by expert qualitative researchers and a GP, then tested with an allied health professional who is also a consumer of general practice. Pilot testing of the interview guide identified the need to better define PCC for participants, which led to the inclusion of the Brickley and colleagues' map for PCC into the interview protocol. Participants will also be provided with the map for PCC prior to the interview. The interview will commence with a short briefing, in which the facilitator will clarify that participants should address the questions on behalf of their practice team and elaborate on any alternate views. Questions will begin with broad inquiry about PCC within the context of the pandemic. Then, participants will be shown the Brickley and colleagues' map for PCC. ${ }^{11}$ Although participants will have already seen the map, participants will be given additional time to reflect on the map and an opportunity to ask questions to clarify their understanding of PCC. Participants will then be asked to elaborate on the strategies their practice has used to deliver the six components of PCC (as outlined in the map) in the context of the pandemic. The interview duration will be a maximum of 30 minutes and allow for probing questions, providing time for interviewees to elaborate on specific concepts and express their views freely. Interview questions will be tailored after the first interview to explore ideas that arose from the initial analyses with subsequent participants, in an iterative process, with developing themes explored in subsequent interviews. ${ }^{19}$

\section{Data collection}

Descriptive practice data collected will include practice names, location (postcode), number of employed full-time equivalent GPs and business model type. The occupation of the practice representative will be recorded. Social demographic and cultural and epidemiological characteristics of the practice catchment area will be collected online from .id (informed decisions) demographic information tools (2020 estimates) and, if recent estimates are not available, from the 2016 national census data (Australian Bureau of Statistics). Interviews will be audio-recorded using a dictaphone or electronically using Microsoft Teams software. Audio-recordings will be subsequently transcribed verbatim using Griffith University's speech-to-text transcription service. All practice representatives must provide verbal consent prior to their interviews. Verbal consent will also be obtained for participating practices to be named in the dissemination of findings. Narrative quotes that could be perceived as sensitive will be sent to interviewees prior to the publication and dissemination of all analyses, as such interviewees will have an opportunity to revise their quotes or withdraw consent to be named. The primary researcher will take field notes 
during all interviews. All participants will be contacted via email and invited to verify the accuracy of their transcripts prior to data analysis.

\section{Data analysis}

Data will be analysed using constant comparison and reflexive thematic analysis, ${ }^{20}$ and this will commence simultaneously with data collection. Field notes, ongoing reflection and debriefing within the research team will support the dependability and credibility of analytical process. ${ }^{21}$ Interview data will be analysed independently by two experienced qualitative researchers. Data will be initially analysed on an individual practice basis, then synthesised and analysed across the whole sample. This meta-synthesis will allow for case comparisons, and the reflective analysis will inform main themes. ${ }^{22}$ Consensus on the analyses of interview data will be sought between two researchers. The analyses will then be shared with the entire research team, who will collaboratively review, define and name themes. ${ }^{21,23}$ Brickley and colleagues' map of PCC will influence the data analysis because it will inform an instrumental definition of PCC. ${ }^{11}$ Practice locations (postcodes) will be analysed by their Accessibility/ Remoteness Index of Australia (ARIA) classification. ${ }^{24}$ The number of employed full-time equivalent GPs will be used to categorise practice size: 1-3 (small), 4-7 (medium) and >7 (large). The analysis of other contextual data (sociocultural) will support the understanding of PCC strategies and perceptions at each practice.

\section{Discussion}

This study will be one of the first to explore the perceptions and experiences of PCC by high-functioning general practice teams throughout the COVID-19 pandemic. Collective case studies help identify views and experiences that may be significantly influenced by contextual factors. This approach is important for the proposed research because the researchers are seeking to identify experiences of PCC during the pandemic and explore if the pandemic has influenced general practice teams' views on the complex concept of PCC. The sampling strategy and collective case method will enable case comparisons of exemplar PCC, which is significant because the subsequent analysis can inform adaptations to policy and practice during the COVID-19 pandemic and beyond.

Limitations of the study design are exacerbated by the need to minimise the burden of participating in research given the clinical demands of participants. Participants will outline the perspectives of PCC on behalf of their practice team, although the ability to capture the practice team's view will be limited as only one team member will be interviewed per practice. Furthermore, the sample consists of general practice staff whose practice had received an award in recognition of quality. The sample is limited to practices who had received an award within the past five years, to strengthen the likelihood that participating practice teams value PCC and can provide examples of delivering PCC under pandemic conditions.

\section{Authors}

Bryce Brickley BNutrDiet (Hons), PhD

Lauren T Williams BSc (Hons), Grad Dip Diet, Grad Dip Soci Sci, PGrad Dip Hlth Prom, PhD, FDA, Head of Nutrition and Dietetics, Griffith University, Qld

Mark Morgan BM, BCh, MA, PhD, MRCGP, FRACGP, Associate Dean for External Engagement, Bond University, Qld

Alyson Ross BAPsych, Post Grad Dip Ed, PhD, EdD, GAICD, Director of Commissioning (Systems), Gold Coast Primary Health Network

Kellie Trigger BA, LLB, Grad Dip Health Economics and Policy, Stakeholder and Engagement Program Manager, Gold Coast Primary Health Network Lauren Ball BAppSc, MNutrDiet, Grad Cert Higher Ed, Grad Dip Health Economics and Health Policy, PhD, Principal Research Fellow, Griffith University, Qld Competing interests: LB is a member of the Australian Journal of General Practice Editorial Advisory Committee, the Dietitians Australia Board and the Griffith University Council, and a committee member of The Royal Australian College of General Practitioners' Handbook of Non-Drug Interventions. LB is Regional Editor of Journal of Human Nutrition \& Dietetics and Associate Editor of Family Practice.

Funding: BB was supported by a PhD scholarship awarded by Griffith University and the Gold Coast Primary Health Network. LB receives a salary from Griffith University and was supported by a National Health and Medical Research Council Fellowship grant (APP1173496). No direct funding was received for this study.

Provenance and peer review: Not commissioned, externally peer reviewed.

Correspondence to:

l.ball@griffith.edu.au

\section{References}

1. World Health Organization. Monitoring the building blocks of health systems: A handbook of indicators and their measurement strategies. Geneva, CH: WHO, 2010.

2. Altin SV, Stock S. The impact of health literacy, patient-centered communication and shared decision-making on patients' satisfaction with care received in German primary care practices. BMC Health Serv Res 2016;16(1):450. doi: 10.1186/ s12913-016-1693-y.

3. Saha S, Beach MC. The impact of patientcentered communication on patients' decision making and evaluations of physicians: A randomized study using video vignettes. Patient Educ Couns 2011;84(3):386-92. doi: 10.1016/j. pec.2011.04.023.

4. Kinmonth AL, Woodcock A, Griffin S, Spiegal N Campbell MJ. Randomised controlled trial of patient centred care of diabetes in general practice: Impact on current wellbeing and future disease risk. The Diabetes Care from Diagnosis Research Team. BMJ 1998;317(7167):1202-08. doi: 10.1136/bmj.317.7167.1202.

5. Egan M, Kessler D, Laporte L, Metcalfe V, Carter M. A pilot randomized controlled trial of community-based occupational therapy in late stroke rehabilitation. Top Stroke Rehabil 2007;14(5):37-45. doi: 10.1310/tsr1405-37.

6. Bauman $A E$, Fardy HJ, Harris PG. Getting it right: Why bother with patient-centred care? Med J Aust 2003;179(5):253-56. doi: 10.5694/j.13265377.2003.tb05532.x.

7. Australian Commission on Safety and Quality in Health Care. National Safety and Quality Health Service Standards. 2nd edn. Sydney, NSW: ACSQHC, 2017.

8. Wright M, Versteeg R, Hall J. General practice's early response to the COVID-19 pandemic. Aust Health Rev 2020;44(5):733-36. doi: 10.1071/ AH20157.

9. POLAR GP. Report into COVID-19 and general practice, insights from the first few weeks. Blackburn East, Vic: Outcome Health, 2020

10. Mira JJ, Blanco M, Cheikh-Moussa K, et al. Proposals for person-centred care in the COVID-19 era. Delphi study. Health Expect 2021;24(2):687-99. doi: 10.1111/hex.13218.

11. Brickley B, Williams LT, Morgan M, Ross A, Trigger K, Ball L. Putting patients first: Development of a patient advocate and general practitioner-informed model of patient-centred care. BMC Health Serv Res 2021;21(1):261. doi: 10.1186/s12913-021-06273-y.

12. The Royal Australian College of General Practitioners. What is General Practice? East Melbourne, Vic: RACGP, 2019. Available at www.racgp.org.au/education/students/a-careerin-general-practice/what-is-general-practice [Accessed 8 June 2020].

13. Australian Government Department of Health Australia's long term national health plan. Canberra, ACT: DoH, 2019.

14. Crotty M. The foundations of social research. Sydney, NSW: Allen \& Unwin, 1998.

15. Sandelowski M. Whatever happened to qualitative description? Res Nurs Health 2000;23(4):334-40. doi: 10.1002/1098-240x(200008)23:4<334::aidnur9>3.0.co;2-g.

16. Brickley B, Sladdin I, Williams $L T$, et al. A new model of patient-centred care for general practitioners: Results of an integrative review. Fam Pract 2020;37(2):154-72. doi: 10.1093/fampra/ cmz063. 
17. Zainal Z. Case study as a research method. Jurnal Kemanusiaan 2007;5(1).

18. Dunham AH, Dunbar JA, Johnson JK, Fuller J, Morgan M, Ford D. What attributions do Australian high-performing general practices make for their success? Applying the clinical microsystems framework: A qualitative study. BMJ Open 2018;8(4):e020552. doi: 10.1136/bmjopen-2017020552.

19. Glaser BG, Strauss AL. Theoretical sampling. In Denzin NK, editor. Sociological methods: A sourcebook. New York, NY: Routledge, 2017; p. 105-14.

20. Braun V, Clarke V. Using thematic analysis in psychology. Qual Res Psychol 2006;3(2):77-101.

21. Creswell JW, Miller DL. Determining validity in qualitative inquiry. Theory Into Practice 2000;39(3):124-30. doi: 10.1207/ s15430421tip3903_2.

22. Walsh D, Downe S. Meta-synthesis method for qualitative research: A literature review. J Adv Nurs 2005;50(2):204-11. doi: 10.1111/j.13652648.2005.03380.x

23. Liamputtong P. Qualitative research methods. 4th edn. South Melbourne, Vic: Oxford University Press, 2013.

24. Queensland Treasury. Accessibility/remoteness index of Australia. Brisbane, QId: Queensland Government, 2019. 